\title{
Axial Angular Momentum of Bessel Light
}

\author{
Mazen S. Nairat* \\ Department of Physics, Al-Balqa Applied University, Al Salt, Jordan
}

Received December 08, 2017; accepted March 14, 2018; published March 31, 2018

\begin{abstract}
Both linear and angular momentum densities of Bessel, Gaussian-Bessel, and Hankel-Bessel lasers are determined. Angular momentum of the three Bessel beams is illustrated at linear and circular polarization. Axial Angular momentum is resolved in particular interpretation: the harmonic order of physical light momentum.
\end{abstract}

Light angular momentum consists of orbital part associated with a helical wave front and spin part associated with polarization. The light of cylindrical symmetric profiles can carry both parts of angular momentum independently. Both angular momentum parts are fundamentally quantized, spin momentum indicating helicity states: left, right handed circular or linear polarization, respectively. Orbital momentum is also quantized per single photon and given by the topological charge of vortex light [1]. Spin and orbital angular momentum of light could be sensed physically by exerted optical torques.

The light of cylindrical axial symmetry is spatially formed in several modes such as Laguerre Gaussian and Bessel light beams. The latter could be formed in three spatial profiles: Bessel, Gaussian-Bessel and HankelBessel beams. Bessel light is used in a wide range of optical applications like communications, tweezers and trapping $[2,3]$.

The three Bessel light modes carry well defined orbital as well as spin angular momentum, they have been mentioned in several studies due to their attractive properties [4, 5]. Extraction of any angular momentum parts of the light could be technically performed, singularity of cylindrical laser beams with different polarization is also investigated [6]. However, it would be worth the effort to provide a general formula of total angular momentum densities for these light modes at momentum states.

This study provides exact analytical expressions of total angular momentum densities of Bessel light at different polarization states. It gives a complete description of the average densities of angular momentum for linear and circular polarization states. Moreover; it resolves direct proportionality of total angular momentum to the main momentum.

*E-mail: mazens@bau.edu.jo
Electromagnetic fields of Bessel's beams are determined at Lorentz gauge using cylindrical coordinates. Although the propagation of Bessel light beams in free space is considered with paraxial approximation, the used analysis could be extended to nonparaxial regime [7]. The obtained results could be generalized for other light beams with cylindrical symmetry, not to mention the enrichment to their optical applications and coding.

The spatial electric field of a linearly polarized beam at Lorentz gauge is given by

$\vec{E}=\left(i \omega u e^{i k z}\right) \hat{/}$ where $u(\rho, \phi, z)$ is field amplitude, $\omega$ is wave frequency and $k$ is the wave number. The polarization is specified by arbitrary axis indicated by arbitrary unit vector $\hat{\prime}$

Linear momentum density for a linearly polarized light is fundamentally given by:

$$
\vec{p}=\frac{\varepsilon}{2}\left(\vec{E}^{*} \times \vec{B}+\vec{E} \times \vec{B}^{*}\right)
$$

Linear momentum density represented in terms of a unit wave amplitude could be represented as [8]:

$$
\vec{p}=\frac{i \omega \varepsilon}{2}\left(u^{*} \nabla u-u \nabla u^{*}\right)+\omega k \varepsilon|u|^{2} \hat{z}
$$

Amplitude of cylindrical symmetric light beams are separable, their wave amplitude split by the exponential of azimuthal angle; $u(\rho, \phi, z)=A(\rho, z) e^{i l \phi}$, where $l$ is the azmithal parameter determining the orbital momentum state. Thus; linear momentum density is rewritten as

$$
\vec{p}=\omega \varepsilon\left\lceil k F \hat{\rho}-\frac{l}{\rho}|A|^{2} \hat{\phi}+k|A|^{2} \hat{z}\right\rceil
$$

where $F=\frac{i}{2 k}\left(A^{*} \frac{\partial A}{\partial \rho}-A \frac{\partial A^{*}}{\partial \rho}\right)$. Angular momentum density is definitely determined by

$$
\vec{l}=\omega \varepsilon\left\lfloor\frac{l z}{\rho} \hat{\rho}-k\left(\rho|A|^{2}-z F\right) \hat{\phi}-l|A|^{2} \hat{z}\right\rfloor
$$

Bessel light (B) is a non-diffracting beam of infinite power. It is physically non-existent even though it is represented by particular wave amplitude as follows [9]:

$$
A_{l}^{B}=C_{B} J_{l}(\kappa \rho) \exp (i k z)
$$

where $C_{B}$ is the normalization constant, $J_{l}$ is the $l$ th order 
Bessel function, $\kappa$ is the transverse component of the wave vector and $l$ is the azimuthal parameter indicating an orbital angular momentum state.

A Bessel light beam is technically modified to generate practical modes which physically available. It could be either modified experimentally to Gaussian-Bessel (GB) [10] or to Hankel-Bessel (HB) [11] modes.

Both Gaussian-Bessel and Hankel-Bessel light carry well-defined orbital angular momentum states independent of a polarization state. Their wave amplitudes in paraxial approximation are respectively represented as follows:

$$
\begin{aligned}
A_{l}^{G B}= & \frac{C_{G B}}{\sqrt{1+\left(z / z_{o}\right)^{2}}} \exp \left\{i\left[\left(k-\frac{\kappa^{2}}{2 k}\right) z-\tan ^{-1}\left(\frac{z}{z_{o}}\right)\right]\right\} J_{I}\left(\frac{\kappa \rho}{1+i z / z_{o}}\right) \\
& \left.\times \exp \left[\rho^{2}\left(\frac{-1}{w^{2}(z)}+\frac{i k}{2 v(z)}\right)\right]\right\rfloor \exp \left[z^{2}\left(\frac{-\kappa}{k^{2} w^{2}(z)}+\frac{i \kappa^{2}}{2 k v(z)}\right)\right]
\end{aligned}
$$

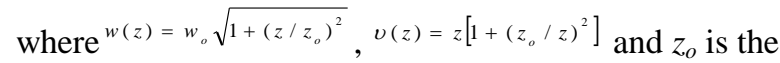
Rayleigh range,

$$
A_{l}^{H B}=C_{H B} H_{/ / 2}^{(1)}\left[\left.k z\left(1-\left(\frac{\rho}{2 z}\right)^{2}\right)\right|_{J / 2}\left(\frac{k \rho^{2}}{4 z}\right)\right.
$$

where ${ }^{H_{1 / 2}^{(1)}}$ is the $l / 2$ th order of Hankel function of first kind. Both linear and angular momentum densities of the three Bessel beams: B, GB and HB are analytically determined for linear and circular polarized cases in the following.

Average linear momentum densities of B, GB and HB beams, using Eq.(3), are respectively given by:

$$
\begin{aligned}
& \vec{p}_{B}=\frac{\left|A_{l}^{B}\right|^{2}}{c}\left(\frac{l}{k \rho} \hat{\phi}+\hat{z}\right) \\
& \vec{p}_{G B}=\frac{\left|A_{l}^{G B}\right|^{2}}{c}\left(\frac{-\rho}{R(z)} \hat{\rho}+\frac{l}{k \rho} \hat{\phi}+\hat{z}\right) \\
& \vec{p}_{H B}=\frac{\left|A_{l}^{H B}\right|^{2}}{c}\left(\frac{l}{k \rho} \hat{\phi}+\hat{z}\right)
\end{aligned}
$$

Linear momentum, which is determined by integration over a beam profile, is only pointed at the axial direction (z) due to symmetry. The other two components: radial and azimuthal vanish. The corresponding angular momentum density using Eq. (4) for B, BG and HB laser beams is given respectively by:

$$
\begin{aligned}
& \vec{\ell}_{B}=\left|A_{l}^{B}\right|^{2}\left(\frac{-l z}{\omega \rho} \hat{\rho}-\frac{\rho}{c} \hat{\phi}+\frac{l}{\omega} \hat{z}\right) \\
& \vec{\ell}_{G B}=\left|A_{l}^{G B}\right|^{2}\left(\frac{-l z}{\omega \rho} \hat{\rho}-\frac{\rho}{c}\left(1+\frac{1}{\left[1+\left(z_{o} / z\right)^{2}\right]}\right) \hat{\phi}+\frac{l}{\omega} \hat{z}\right)
\end{aligned}
$$

$$
\vec{\ell}_{H B}=\left|A_{l}^{H B}\right|^{2}\left(\frac{-l z}{\omega \rho} \hat{\rho}-\frac{\rho}{c} \hat{\phi}+\frac{l}{\omega} \hat{z}\right)
$$

Angular momentum of the laser beams $(\vec{L})$, which is integration of angular momentum density over the beam profile, is also reduced to only an axial component.

Unlike a plane wave beam, angular momentum of a symmetric cylindrical light beam is along the propagation axis. However; there is a transverse component of the pointing vector of such light beams causing azimuthal spiraling of their wave fronts.

Both linear momentum and angular momentum for $B, G B$, and $H B$ are axially oriented, they are directly proportional for each single beam, i.e:

$L=\int \ell d^{3} \rho=\frac{l}{k} \int p d^{3} \rho=\frac{l}{k} P$

Normalization of B, GB and HB beam profiles have been considered.

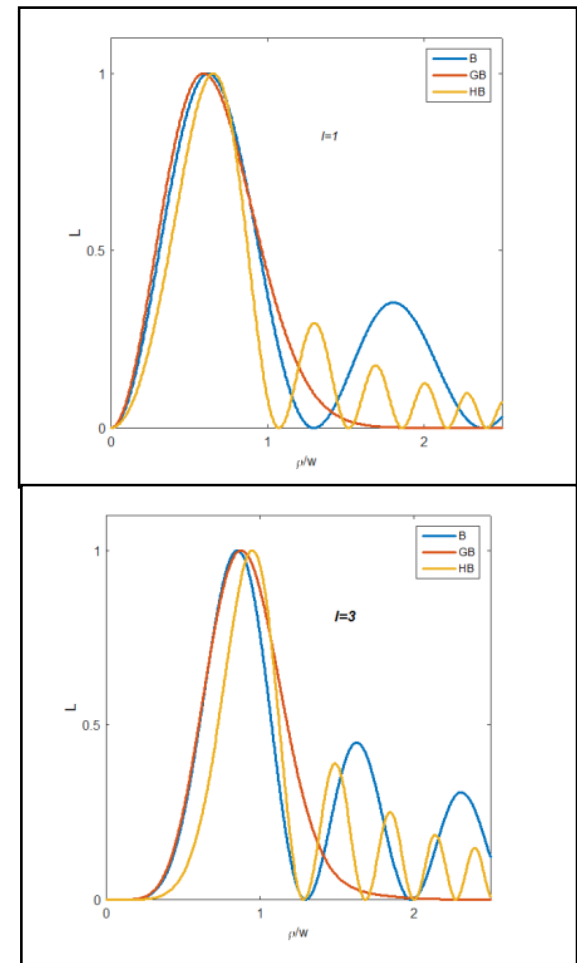

Fig 1. Axial angular momentum of Bessel beams at orbital angular momentum states $l=1$ and 2 , respectively.

Equation (10) has been mentioned before for another cylindrical symmetric light beam [8] but without a convenient physical meaning. This study proposes a resolution of axial angular momentum of cylindrically symmetrical light as is a harmonic order of its linear momentum. The harmonic order of light momentum is indicated by a well-defined angular momentum state $l$. Unlike linear momentum, the angular momentum profile, 
which is characterized by the azmithual mode $l$, indicates the orientation of energy flux. Their intensity is visualized as coaxial rings.

Axial angular momenta of the three Bessel beams are computed at different orbital states, the numerical results are plotted in Fig.1 versus normalized beam waist, $\rho / w$. Figure 1 shows the distribution of angular momentum over Bessel beams profiles, the change in the orbital state $(l)$ indicates a harmonic order of beams fluxes.

The linear momentum density, Eq. (3), of a circular polarized light beam is modified to include a polarization dependent term. It is given by [12]:

$$
\left.\vec{p}=\left.\omega\left|k F \hat{\rho}-\left(\frac{l}{\rho}|A|^{2}+\frac{\sigma}{2} \frac{\partial|A|^{2}}{\partial \rho}\right) \hat{\phi}+k\right| A\right|^{2} \hat{z}\right]
$$

where $\sigma=\mp 1$ for the right-hand or left-hand circular polarization, respectively.

Associated angular momentum densities of circular polarized B, GB and HB beam are given respectively by:

$$
\begin{gathered}
\overrightarrow{\mathfrak{I}}_{B}=\omega\left|A_{l}^{B}\right|^{2}\left[z\left(\frac{l}{\rho}-\sigma R^{B}\right) \hat{\rho}-k \rho \hat{\phi}+\left(l-\sigma R^{B}\right) \hat{z}\right] \\
\overrightarrow{\mathfrak{I}}_{G B}=\omega\left|A_{l}^{G B}\right|^{2}\left[k\left(\frac{l}{\rho}-\sigma R^{G B}\right) \hat{\rho}-k \rho\left(1+\frac{1}{1+i z / z_{o}}\right) \hat{\phi}+\left(l-\sigma R^{G B}\right) \hat{z}\right] \\
\overrightarrow{\mathfrak{J}}_{H B}=\omega\left|A_{l}^{H B}\right|^{2}\left[k\left(\frac{l}{\rho}-\sigma R^{H B}\right) \hat{\rho}-k \rho \hat{\phi}+\left(l-\sigma R^{H B}\right) \hat{z}\right]
\end{gathered}
$$

where

$$
\begin{aligned}
& R^{B}=\frac{1}{2} \frac{\partial}{\partial \rho} \ln \left|J_{1}(\kappa \rho)\right|, \\
& R^{G B}=\frac{\rho}{w^{2}(z)}+\frac{1}{2} \frac{\partial}{\partial \rho} \ln \left|J_{l}\left(\frac{\kappa \rho}{1+i z / z_{o}}\right)\right| \\
& R^{H B}=\frac{1}{2} \frac{\partial}{\partial \rho} \ln \left|H_{L / 2}^{(1)}\left[k z\left(1+(\rho / z)^{2}\right)\right] J_{l / 2}\left(\frac{k \rho^{2}}{4 z}\right)\right|
\end{aligned}
$$

Total angular momentum is reduced again to only axial, $z$, component due to symmetry, it is pointed to the same orientation of linear momentum too. Axial angular momentum for circularly polarized B, GB, HB light beams is determined by the following ratio:

$$
J=\int \mathfrak{J} d^{3} \rho=\frac{l+\sigma}{k} \int p d^{3} \rho=\frac{l+\sigma}{k} P
$$

where the integration of angular momentum density is only performed over the $\mathrm{z}$ component of Eqs. (12). The proportionality in Eq.(13) satisfies B, GB and HB light beams, however, it is evaluated in details only for GB mode, a similar technique is applicable for both $\mathrm{H}$ and $\mathrm{HB}$ as well.

$$
\begin{aligned}
& \frac{\int \mathfrak{J}_{G B} d^{3} \rho}{\int p_{G B} d^{3} \rho}=\frac{\int\left|u_{G B}\right|^{2}\left(l-\sigma \rho R^{G B}\right) d^{3} \rho}{\int\left|u_{G B}\right|^{2} d^{3} \rho} \\
& =\frac{\int\left|u_{G B}\right|^{2}\left(l-\sigma \rho R^{G B}\right) \rho d \rho}{\int\left|u_{G B}\right|^{2} \rho d \rho} \\
& =l-\sigma \int\left|u_{G B}\right|^{2} \rho^{2} R^{G B} d \rho \\
& =l-\frac{\sigma}{2} \int \rho^{2} \frac{\partial\left|u_{G B}\right|^{2}}{\partial \rho} d \rho=\frac{l+\sigma}{k}
\end{aligned}
$$

Equation (13) confirms the axial angular momentum of Bessel light at circular polarization. Axial angular momentum is interpreted as a harmonic order of physical light momentum pointed along the propagation axis. Moreover, Eq. (13) is not only compatible with the ratio derived for Laguerre-Gaussian light, as shown in [8], but also compatible with any cylindrical symmetric light.

In conclusion, this study provides analytical expressions for linear and angular momentum densities for Bessel beams: B, GB, and HB. Bessel light beams are considered at linear as well as circular polarization. Axial angular momentum has been resolved as an interpretation of physical light momentum. It is just a harmonic order of physical light momentum. This result is applicable not only for Bessel light but also for an arbitrary light beam of cylindrical symmetry

\section{References}

[1] G. Molina-Terriza, J. Torres, L. Torner, Nature Physics 3, 305 (2007)

[2] J. Arlt, V. Garces-Chavez, W. Sibbett, K. Dholakia, Opt. Commun. 197, 4 (2001).

[3] L. Ambrosio, H. Hernández-Figueroa, Opt. Express 18, 23 (2010)

[4] I. Litvin, A. Dudley, A. Forbes, Opt. Express 19, 18 (2011).

[5] K. Volke-Sepulveda, V. Garcés-Chávez, S. Chávez-Cerda, J. Arlt, K. Dholakia, J. Opt. B 4 (2), S82 (2002).

[6] M. Verma, S. Pal, S. Joshi, P. Senthilkumaran, J. Joseph, H Kandpal, J. Mod. Opt. 62 (13), 1068 (2015)

[7] R. Borghi, M. Santarsiero, M. Porras, J. Opt. Soc. Am. A, 18 (7), 2100 (2011).

[8] L. Allen, M. Beijersbergen, R. Spreeuw, J. Woerdman, Phys. Rev. A 45(11), 8185 (1992).

[9] D. Mcglion, K. Dholakia, Cont. Phys. 46(1), 15 (2005)

[10] F. Gori, G. Guattari, C. Padovani, Opt. Commun. 64, 491 (1987).

[11] V. Kotlyar, A. Kovalev, A. Soifer, J. Opt. Soc. Am. A, 29 (5), 741 (2012).

[12] L. Allen, M. Babiker, Phys. Rev. A 53, R2937 (1996). 